\title{
Born effective charge removed anomalous temperature dependence of lattice thermal conductivity in monolayer GeC
}

\author{
San-Dong Guo \\ School of Physics, China University of Mining and Technology, Xuzhou 221116, Jiangsu, China
}

\begin{abstract}
Due to potential applications in nano- and opto-electronics, two-dimensional (2D) materials have attracted tremendous interest. Their thermal transport properties are closely related to the performance of 2D materials-based devices. Here, the phonon transports of monolayer GeC with a perfect planar hexagonal honeycomb structure are investigated by solving the linearized phonon Boltzmann equation within the single-mode relaxation time approximation (RTA). Without inclusion of Born effective charges $\left(Z^{*}\right)$ and dielectric constants $(\varepsilon)$, the lattice thermal conductivity $\left(\kappa_{L}\right)$ almost decreases linearly above $350 \mathrm{~K}$, deviating from the usual $\kappa_{L} \sim 1 / T$ law. The underlying mechanism is because the contribution to $\kappa_{L}$ from high-frequency optical phonon modes increases with increasing temperature, and the contribution exceeds one from acoustic branches at high temperature. These can be understood by huge phonon band gap caused by large difference in atom mass between Ge and $\mathrm{C}$ atoms, which produces important effects on scattering process involving high-frequency optical phonon. When considering $Z^{*}$ and $\varepsilon$, the phonon group velocities and phonon lifetimes of high-frequency optical phonon modes are obviously reduced with respect to ones without $Z^{*}$ and $\varepsilon$. The reduced group velocities and phonon lifetimes give rise to small contribution to $\kappa_{L}$ from highfrequency optical phonon modes, which produces the the traditional $\kappa_{L} \sim 1 / T$ relation in monolayer GeC. Calculated results show that the isotope scattering can also reduce anomalous temperature dependence of $\kappa_{L}$ in monolayer GeC. Our works highlight the importance of $Z^{*}$ and $\varepsilon$ to investigate phonon transports of monolayer $\mathrm{GeC}$, and motivate further theoretical or experimental efforts to investigate thermal transports of other $2 \mathrm{D}$ materials.
\end{abstract}

PACS numbers: 72.15.Jf, 71.20.-b, 71.70.Ej, 79.10.-n Keywords: Lattice thermal conductivity; Monolayer; Born effective charge

Email:sandongyuwang@163.com

\section{INTRODUCTION}

Since the successful synthesis of graphene ${ }^{1}, 2 \mathrm{D}$ materials have attracted increasing attention due to potential applications in electronics, spintronics and optoelectronics $^{2-9}$. Thermal management is a significant factor for these applications ${ }^{10}$. To efficiently dissipate heat in electronic devices, a high thermal conductivity is required, while a low lattice thermal conductivity is beneficial to thermoelectric applications, to achieve good thermoelectric performance ${ }^{11,12}$. Diverse anisotropy of phonon transport in group IV-VI monolayer is predicted by solving the Boltzmann transport equation ${ }^{13}$. Phonon transport properties of group-IV and -VA element monolayers have been performed theoretically ${ }^{14-16}$. The $\kappa_{L}$ of transition metal dichalcogenide (TMD) and Janus TMD monolayers have been systematically studied by phonon Boltzmann transport equation approach ${ }^{17,18}$. Strain effects on thermal transports of Sb monolayer ${ }^{19}$, group-IV monolayers $^{20}$ and $2 \mathrm{D}$ penta-structures materials ${ }^{21}$ have also been studied, showing diverse strain dependence, such as monotonously increasing or decreasing and upand-down behaviors with increasing tensile strain.

For most bulk and 2D materials, the temperaturedependent $\kappa_{L}$ follows the relation of $\kappa_{L} \sim 1 / T$. Recently, anomalously temperature-dependent $\kappa_{L}$ of monolayer $\mathrm{ZnO}$ and $\mathrm{GaN}$ is predicted by a first-principles study ${ }^{22,23}$, which is due to the huge phonon band gap in their phonon dispersions. The predicted room-temperature $\kappa_{L}$ of monolayer $\mathrm{ZnO}$ and $\mathrm{GaN}$ is $4.5 \mathrm{Wm}^{-1} \mathrm{~K}^{-1}$ with

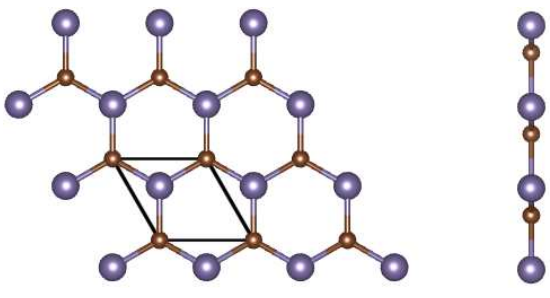

FIG. 1. (Color online) The top (Left) and side (Right) view of monolayer $\mathrm{GeC}$, and the frame surrounded by a black box is unit cell. The large and small balls represent Ge and $\mathrm{C}$ atoms, respectively.

the thickness of $3.04 \AA$ and $14.93 \mathrm{Wm}^{-1} \mathrm{~K}^{-1}$ with the thickness of $3.74 \AA$, respectively. The $\mathrm{SiC}$ monolayer has the same perfect planar hexagonal honeycomb structure with $\mathrm{ZnO}$ and $\mathrm{GaN}$, but the $\kappa_{L}$ of $\mathrm{SiC}$ monolayer follows the conventional $1 / T$ law $^{24}$, which may be due to small phonon band gap. For GeC monolayer with the same structure, a large phonon band gap can be observed due to a large difference in atom mass between Ge and $\mathrm{C}$ atoms $^{25}$. The similar anomalous temperature dependence of $\kappa_{L}$ may also exist in monolayer GeC. In this work, based on first-principles calculations, the phonon transport properties of monolayer $\mathrm{GeC}$ are investigated by solving the linearized phonon Boltzmann equation. When neglecting $Z^{*}$ and $\varepsilon$, the $\kappa_{L}$ deviates from the usual $\kappa_{L} \sim 1 / T$ law. The $\kappa_{L}$ above $200 \mathrm{~K}$ is much higher than the expected $\kappa_{L}$ predicted from the general $\kappa_{L} \sim 1 / T$ law. The large deviation stems from the high-frequency opti- 

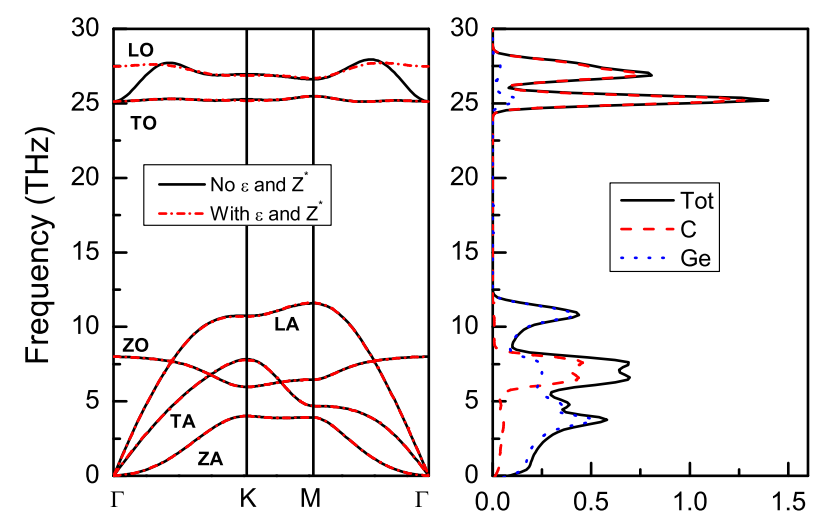

FIG. 2. (Color online) Phonon band structures of monolayer GeC with or without $\varepsilon$ and $Z^{*}$, along with the total and partial DOS without $\varepsilon$ and $Z^{*}$.

cal phonon modes, whose contribution to $\kappa_{L}$ increases with increasing temperature, and eventually dominates $\kappa_{L}$. With inclusion of $Z^{*}$ and $\varepsilon$, the phonon group velocities and phonon lifetimes of high-frequency optical phonon modes are obviously reduced, which gives rise to small contribution to $\kappa_{L}$ from high-frequency optical phonon modes, producing the the traditional $\kappa_{L} \sim 1 / T$ relation in monolayer $\mathrm{GeC}$. It is found that the isotope scattering can also reduce anomalous temperature dependence of $\kappa_{L}$ in monolayer GeC.

The rest of the paper is organized as follows. In the next section, we shall give our computational details about phonon transport. In the third section, we shall present phonon transport of monolayer GeC. Finally, we shall give our discussion and conclusions in the fourth section.

\section{COMPUTATIONAL DETAIL}

All first-principles calculations are carried out based on the density functional theory (DFT) using the projected augmented wave (PAW) method, and the generalized gradient approximation of the Perdew-Burke-Ernzerhof (GGA-PBE) is adopted as exchange-correlation energy functional, as implemented in the Vienna ab initio simulation package (VASP) ${ }^{26-29}$. A plane-wave basis set is employed with kinetic energy cutoff of $700 \mathrm{eV}$, and the $2 s 2 p(4 s 4 p)$ orbitals of $\mathrm{C}(\mathrm{Ge})$ atoms are treated as valance ones. To avoid spurious interaction, the unit cell of monolayer $\mathrm{GeC}$ is built with the vacuum region of $18 \AA$ along the out-of-plane direction. The energy convergence threshold is set as $10^{-8} \mathrm{eV}$.

The $\kappa_{L}$ of monolayer GeC is calculated by solving linearized phonon Boltzmann equation with the single mode RTA, as implemented in the Phono3py code ${ }^{30}$. The $\kappa_{L}$ can be expressed as:

$$
\kappa=\frac{1}{N V_{0}} \sum_{\lambda} \kappa_{\lambda}=\frac{1}{N V_{0}} \sum_{\lambda} C_{\lambda} \nu_{\lambda} \otimes \nu_{\lambda} \tau_{\lambda}
$$

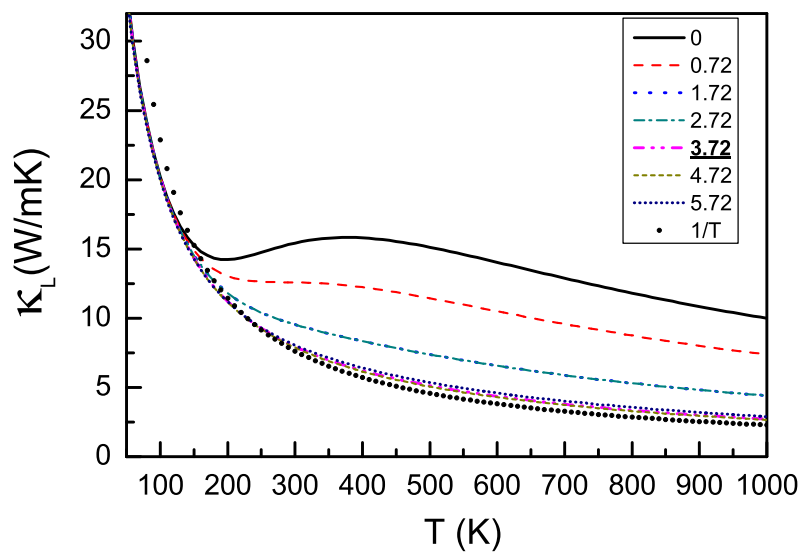

FIG. 3. (Color online) The $\kappa_{L}$ of monolayer GeC with or without $\varepsilon$ and $Z^{*}$ as a function of temperature (3.72 and 0 ); The $\kappa_{L} \sim 1 / T$ relation is plotted for comparison; The $\kappa_{L}$ with $\left|Z^{*}\right|$ along $x x$ and $y y$ directions artificially changing from 0.72 to 5.72 .

where $\lambda, N$ and $V_{0}$ are phonon mode, the total number of q points sampling Brillouin zone (BZ) and the volume of a unit cell, and $C_{\lambda}, \nu_{\lambda}, \tau_{\lambda}$ is the specific heat, phonon velocity, phonon lifetime. The phonon lifetime $\tau_{\lambda}$ can be attained by phonon linewidth $2 \Gamma_{\lambda}\left(\omega_{\lambda}\right)$ of the phonon mode $\lambda$ :

$$
\tau_{\lambda}=\frac{1}{2 \Gamma_{\lambda}\left(\omega_{\lambda}\right)}
$$

The $\Gamma_{\lambda}(\omega)$ takes the form analogous to the Fermi golden rule:

$$
\begin{gathered}
\Gamma_{\lambda}(\omega)=\frac{18 \pi}{\hbar^{2}} \sum_{\lambda^{\prime} \lambda^{\prime \prime}}\left|\Phi_{-\lambda \lambda^{\prime} \lambda^{\prime \prime}}\right|^{2}\left[( f _ { \lambda } ^ { \prime } + f _ { \lambda } ^ { \prime \prime } + 1 ) \delta \left(\omega-\omega_{\lambda}^{\prime}-\right.\right. \\
\left.\left.\omega_{\lambda}^{\prime \prime}\right)+\left(f_{\lambda}^{\prime}-f_{\lambda}^{\prime \prime}\right)\left[\delta\left(\omega+\omega_{\lambda}^{\prime}-\omega_{\lambda}^{\prime \prime}\right)-\delta\left(\omega-\omega_{\lambda}^{\prime}+\omega_{\lambda}^{\prime \prime}\right)\right]\right]
\end{gathered}
$$

in which $f_{\lambda}$ and $\Phi_{-\lambda \lambda^{\prime} \lambda^{\prime \prime}}$ are the phonon equilibrium occupancy and the strength of interaction among the three phonons $\lambda, \lambda^{\prime}$, and $\lambda^{\prime \prime}$ involved in the scattering.

The interatomic force constants (IFCs) are calculated by the finite displacement method. The second-order harmonic (third-order anharmonic) IFCs are calculated using a $5 \times 5 \times 1(4 \times 4 \times 1)$ supercell containing 50 (32) atoms with $\mathrm{k}$-point meshes of $4 \times 4 \times 1$. Using the harmonic IFCs, phonon dispersion of monolayer GeC can be attained, using Phonopy package ${ }^{31}$. To compute lattice thermal conductivities, the reciprocal spaces of the primitive cells are sampled using the $100 \times 100 \times 1$ meshes. For 2D material, the calculated lattice thermal conductivity depends on the length of unit cell used in the calculations along $z$ direction $^{32}$. The lattice thermal conductivity should be normalized by multiplying $L z / d$, in which $L z$ is the length of unit cell along $z$ direction and $d$ is the thickness of $2 \mathrm{D}$ material, but the $d$ is not well defined like graphene. In this work, the length of unit cell (18 $\AA$ ) along $\mathrm{z}$ direction is used as the thickness 


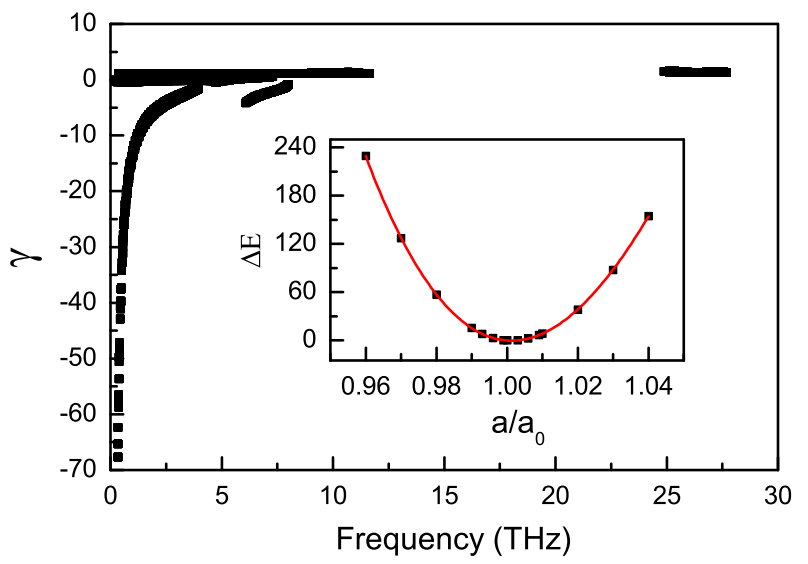

FIG. 4. (Color online) The mode level Grüneisen parameters of monolayer $\mathrm{GeC}$ in the first BZ; (Inset) the potential energy well $\Delta E=E_{a / a_{0}}-E_{1}$ as a function of $a / a_{0}$.

of monolayer GeC. To make a fair comparison between various $2 \mathrm{D}$ monolayers, the thermal sheet conductance can be used, defined as $\kappa_{L} \times d$.

\section{MAIN CALCULATED RESULTS AND ANALYSIS}

Monolayer $\mathrm{GeC}$ prefers a perfect planar hexagonal honeycomb structure, and similar monolayer structure can be found in graphene, $\mathrm{ZnO}, \mathrm{GaN}$ and $\mathrm{SiC}^{22,23,25}$. The monolayer $\mathrm{GeC}$ can be built by replacing one atom in the unit cell of graphene with Ge atom, and the space symmetry group is $P \overline{6} M 2$, being lower than that of graphene $(P 6 / M M M)$. Figure 1 shows the schematic crystal structure of monolayer $\mathrm{GeC}$, and the optimized lattice parameter within GGA-PBE is $3.26 \AA$. Firstly, the elastic properties of monolayer $\mathrm{GeC}$ are studied, and two independent elastic constants $C_{11}\left(=C_{22}\right)$ and $C_{12}$ due to $D_{3 h}$ symmetry can be calculated, and the $C_{66}=\left(C_{11^{-}}\right.$$\left.C_{12}\right) / 2$. For $C_{11}, C_{12}$ and $C_{66}$, the calculated value is $159.42 \mathrm{Nm}^{-1}, 51.62 \mathrm{Nm}^{-1}$ and $53.90 \mathrm{Nm}^{-1}$, respectively. These $C_{i j}$ satisfy the Born criteria of mechanical stability. Based on calculated $C_{i j}$, the $2 \mathrm{D}$ Youngs moduli $Y^{2 D}$ and shear modulus $G^{2 D}$ of monolayer $\mathrm{GeC}^{33}$ are 142.71 $\mathrm{Nm}^{-1}$ and $53.90 \mathrm{Nm}^{-1}$, which are lower than ones of graphene and $\mathrm{SiC}$ monolayer ${ }^{24,33}$. The $\mathrm{GeC}$ monolayer is more flexible than graphene and $\mathrm{SiC}$ monolayer due to smaller $Y^{2 D}$.

TABLE I. The $Z^{*}$ of $\mathrm{C}$ and Ge atoms and $\varepsilon$ of monolayer GeC. Except for $x x, y y$ and $z z$ directions, the $Z^{*}$ and $\varepsilon$ along other directions are zero.

\begin{tabular}{cccc}
\hline \hline Direction & $Z^{*}(\mathrm{C})$ & $Z^{*}(\mathrm{Ge})$ & $\varepsilon$ \\
\hline \hline$x x$ & -3.72 & 3.72 & 2.90 \\
$y y$ & -3.72 & 3.72 & 2.90 \\
$z z$ & -0.25 & 0.25 & 1.18 \\
\hline \hline
\end{tabular}
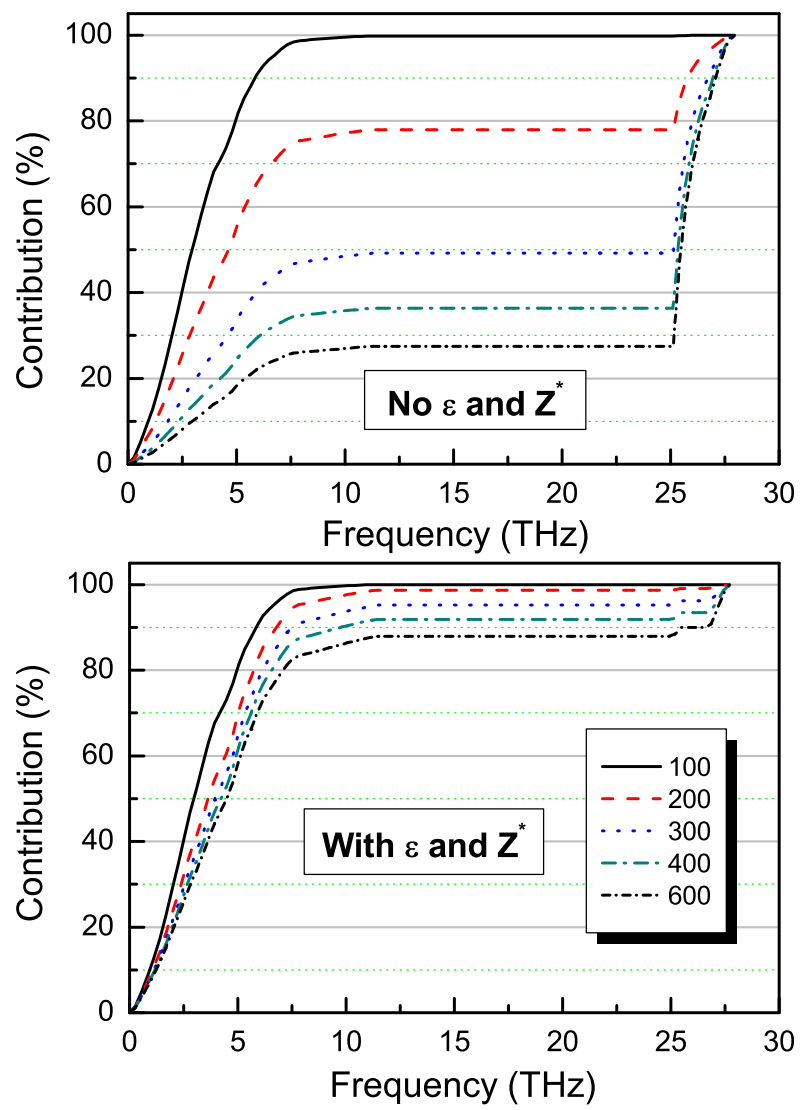

FIG. 5. (Color online) At 100, 200, 300, 400 and $600 \mathrm{~K}$, the ratio between accumulated and total $\kappa_{L}$ with respect to frequency with or without $\varepsilon$ and $Z^{*}$.

The calculated phonon dispersion of monolayer GeC along high-symmetry path and total and partial density of states (DOS) are shown in Figure 2. No imaginary frequencies are observed in the phonon dispersion, indicating the thermodynamic stability of monolayer GeC. There are 6 phonon branches due to 2 atoms per unit cell, including 3 acoustic and 3 optical phonon branches. A phonon band gap of $13.52 \mathrm{THz}$ is observed, which separates in-plane transverse optical (TO) and the in-plane longitudinal optical (LO) branches from out-of-plane optical (ZO), in-plane longitudinal acoustic (LA), in-plane transverse acoustic (TA) and out-of-plane acoustic (ZA) branches. It is noted that the phonon band gap is larger than width of acoustic branches $(11.61 \mathrm{THz})$, which has important effects on phonon transport. The large gap can be explained by the Ge atom being much heavier than $\mathrm{C}$ atom. It is clearly seen that the $\mathrm{ZO}$ branch crosses with the TA and LA branches, which has significant effect on the phonon scattering process. The similar phonon dispersion can also be found in $\mathrm{SiC}, \mathrm{ZnO}$ and GaN monolayers ${ }^{22-25}$. However, for monolayer $\mathrm{SiC}$, a phonon band gap of $7.47 \mathrm{THz}$ is very smaller than width of acoustic branches (19.48 THz). Based on the elastic theory, the ZA phonon branch should have quadratic dispersion with the sheet being free of stress $^{34,35}$. The 


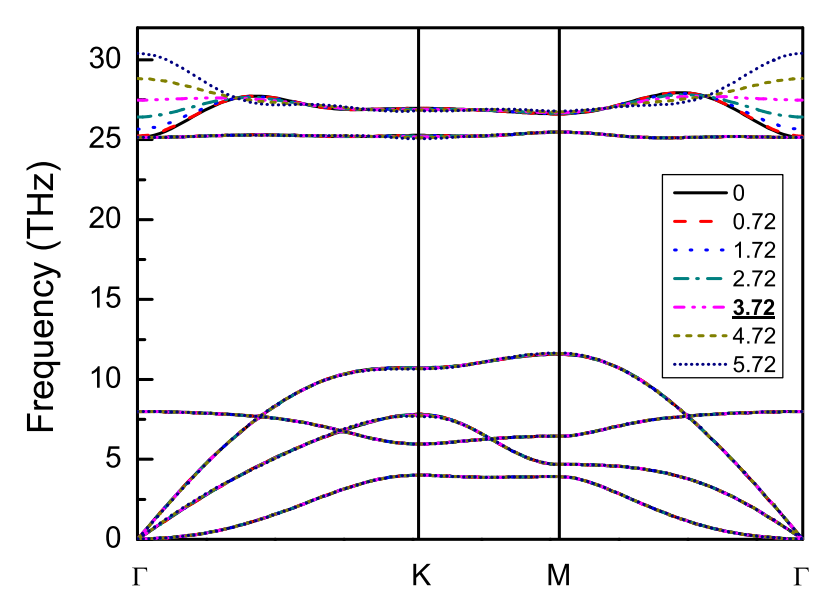

FIG. 6. (Color online) Phonon band structures of monolayer $\mathrm{GeC}$ with $\left|Z^{*}\right|$ along $x x$ and $y y$ directions artificially changing from 0 to 5.72 .

quadratic ZA branch near the $\Gamma$ point can be observed for monolayer GeC. However, the TA and LA branches are linear near the $\Gamma$ point. The partial DOS indicates that optical branches are mainly contributed by the vibrations of $\mathrm{C}$ atoms, while acoustic branches are contributed by the vibrations of Ge atoms.

By solving the linearized phonon Boltzmann equation within single-mode RTA method, the intrinsic $\kappa_{L}$ of monolayer GeC is calculated, which is plotted in Figure 3 as a function of temperature. The room-temperature $\kappa_{L}$ of monolayer $\mathrm{GeC}$ is $15.43 \mathrm{Wm}^{-1} \mathrm{~K}^{-1}$ with the thickness of $18 \AA$, and the corresponding thermal sheet conductance is $277.74 \mathrm{WK}^{-1}$, being two orders of magnitude lower than that of graphene (about $\left.12884 \mathrm{WK}^{-1}\right)^{32}$. To understand the mechanism underlying the low $\kappa_{L}$ of monolayer GeC, the mode level Grüneisen parameters of monolayer $\mathrm{GeC}$ are show in Figure 4. It is found that the $\gamma$ of TO and LO branches is fully positive. For the phonon modes below the gap, both negative and partial positive $\gamma$ can be observed, and ZA branch shows very large negative $\gamma$. However, due to the symmetry-based selection rule, the scattering of ZA branche is largely suppressed. The large $\gamma$ means strong phonon anharmonicity, which can produce the strong phonon-phonon scattering, leading to the low $\kappa_{L}$ of monolayer GeC. To have an explicit view on the phonon anharmonicity from another aspect, the potential energy well of monolayer GeC, defined as potential energy change due to the change of lattice constant $\left(\Delta E=E_{a / a_{0}}-E_{1}\right.$, where $E_{a / a_{0}}$ and $E_{1}$ are the total energies of strained and unstrained systems, respectively), is shown in the inset of Figure 4. It is found that the potential well of monolayer $\mathrm{GeC}$ is asymmetric with respect to compressive and tensile strains, which is a direct evidence of the phonon anharmonicity. A three order polynomial curve can be used to fit the potential energy well, and the fitted parameter for the cubic term is $-395 \mathrm{eV}$, and the large cubic term is consistent with the $\gamma$.
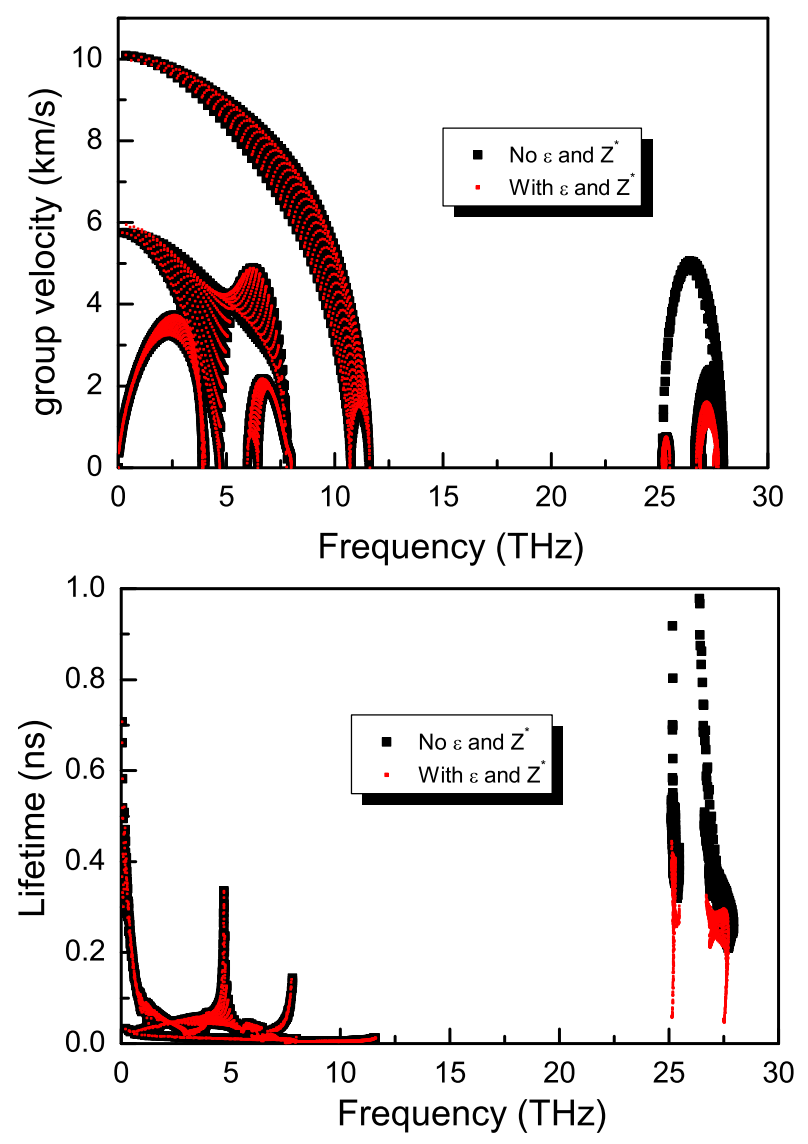

FIG. 7. (Color online) The mode level phonon group velocities and phonon lifetimes $(300 \mathrm{~K})$ of monolayer $\mathrm{GeC}$ in the first BZ with or without $\varepsilon$ and $Z^{*}$.

For the most bulk and 2D materials, the temperaturedependent $\kappa_{L}$ follows $\kappa_{L} \sim 1 / T^{\alpha}$ relationship with $\alpha$ changing from 0.85 to $1.05^{22}$. It is very strange that the $\kappa_{L}$ of monolayer GeC shows an anomalous linear temperature dependence above about $350 \mathrm{~K}$, which is obviously different from usual picture $\kappa_{L} \sim 1 / T$ relationship. The $\kappa_{L} \sim 1 / T$ relation is also shown in Figure 3 for comparison. To understand the anomalous temperature dependence of $\kappa_{L}$ of monolayer $\mathrm{GeC}$, the ratio between accumulated and total $\kappa_{L}$ with respect to frequency at 100, 200, 300, 400 and $600 \mathrm{~K}$ are plotted in Figure 5. It is clearly seen that, with the temperature increasing, the contribution from LO and TO branches increases. When the temperatures are lower than $300 \mathrm{~K}$, the contribution of phonon modes below the phonon gap is larger than $50 \%$. However, for temperatures higher than $300 \mathrm{~K}$, the LO and TO branches dominate the $\kappa_{L}$ with the contribution larger than 50\%. If acoustic phonon branches dominate $\kappa_{L}$, the temperature-dependent $\kappa_{L}$ would follow $\kappa_{L} \sim 1 / T$ relationship, which can be found for most materials $^{22}$. So, the anomalous linear temperature dependence of $\kappa_{L}$ of monolayer $\mathrm{GeC}$ is due to dominant contribution from LO and TO branches, when the temperature is larger than $300 \mathrm{~K}$. Anomalously temperature- 


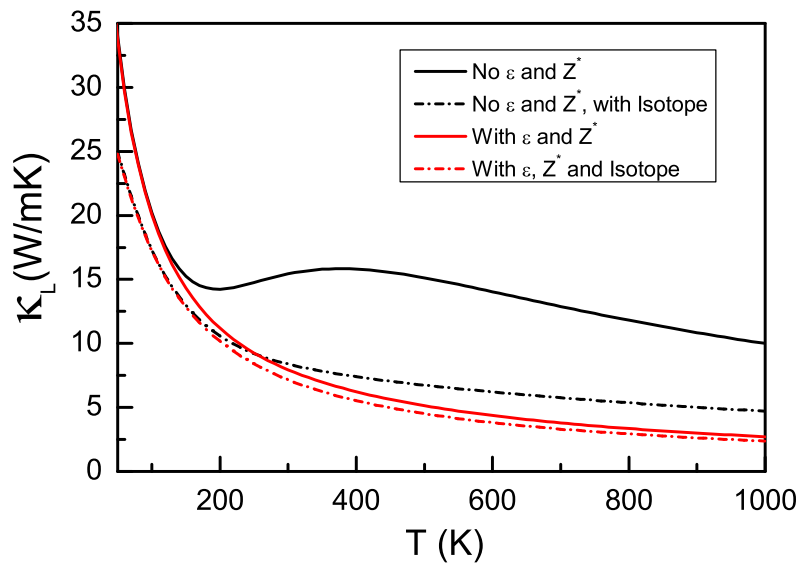

FIG. 8. (Color online) The $\kappa_{L}$ of monolayer GeC with or without $\varepsilon, Z^{*}$ and isotope as a function of temperature.

dependent $\kappa_{L}$ has been predicted in monolayer $\mathrm{ZnO}$ and $\mathrm{GaN}$ by $\mathrm{Hu}$ et al. ${ }^{22}$. To achieve the linear temperature dependence of $\kappa_{L}$, Hu et al. propose two conditions: (1) With increased temperature, the contribution to $\kappa_{L}$ from optical phonon branches should increases. (2) At high temperature, the optical branches should dominate $\kappa_{L}$. The monolayer $\mathrm{GeC}$ indeed satisfies these conditions.

Due to the large charge transfer from Ge to C atom, the long-range electrostatic Coulomb interactions may have important effects on phonon transports of monolayer GeC. The large charge transfer can induce strongly polarized covalent bond, which is also described by $Z^{*}$ and $\varepsilon$, as given in Table I. The large $Z^{*}$ and $\varepsilon$ can produce large LO-TO splitting $(2.35 \mathrm{THz})$ at the $\mathrm{BZ}$ center, as seen in Figure 2. The $\kappa_{L}$ with $Z^{*}$ and $\varepsilon$ as a function of temperature is also plotted in Figure 3 . It is clearly seen that the relationship between $\kappa_{L}$ and $T$ changes from $\kappa_{L} \sim T$ to $\kappa_{L} \sim 1 / T$. To understand the sudden change, the ratio between accumulated and total $\kappa_{L}$ with respect to frequency with $Z^{*}$ and $\varepsilon$ are also plotted in Figure 5 at $100,200,300,400$ and $600 \mathrm{~K}$. With increased temperature, the contribution to $\kappa_{L}$ from high frequency optical phonon branches increases, but acoustic branches always dominate the $\kappa_{L}$ for all temperatures $(>80 \%)$. With inclusion of $Z^{*}$ and $\varepsilon$, the condition (2) is broken, so the $\kappa_{L} \sim T$ relationship disappears.

To have a better understanding about $Z^{*}$ effects on $\kappa_{L}$, we artificially change $\left|Z^{*}\right|$ along $x x$ and yy directions from 0 to 5.72 , and the related phonon dispersions and $\kappa_{L}$ are plotted in Figure 6 and Figure 3, respectively. The LO-TO splitting changes from $0 \mathrm{THz}$ to $5.17 \mathrm{THz}$ with increasing $\left|Z^{*}\right|$, and the $\kappa_{L}$ gradually deviates from the $\kappa_{L} \sim T$ relationship. When $\left|Z^{*}\right|$ reaches the true values, $\kappa_{L} \sim 1 / T$ can be observed. To understand the mechanism underlying the sudden change of $\kappa_{L}$, the mode level phonon group velocities and phonon lifetimes (300K) of monolayer $\mathrm{GeC}$ with or without $\varepsilon$ and $Z^{*}$ are plotted in Figure 7. The group velocities and phonon lifetimes of phonon modes below the gap have little difference be-

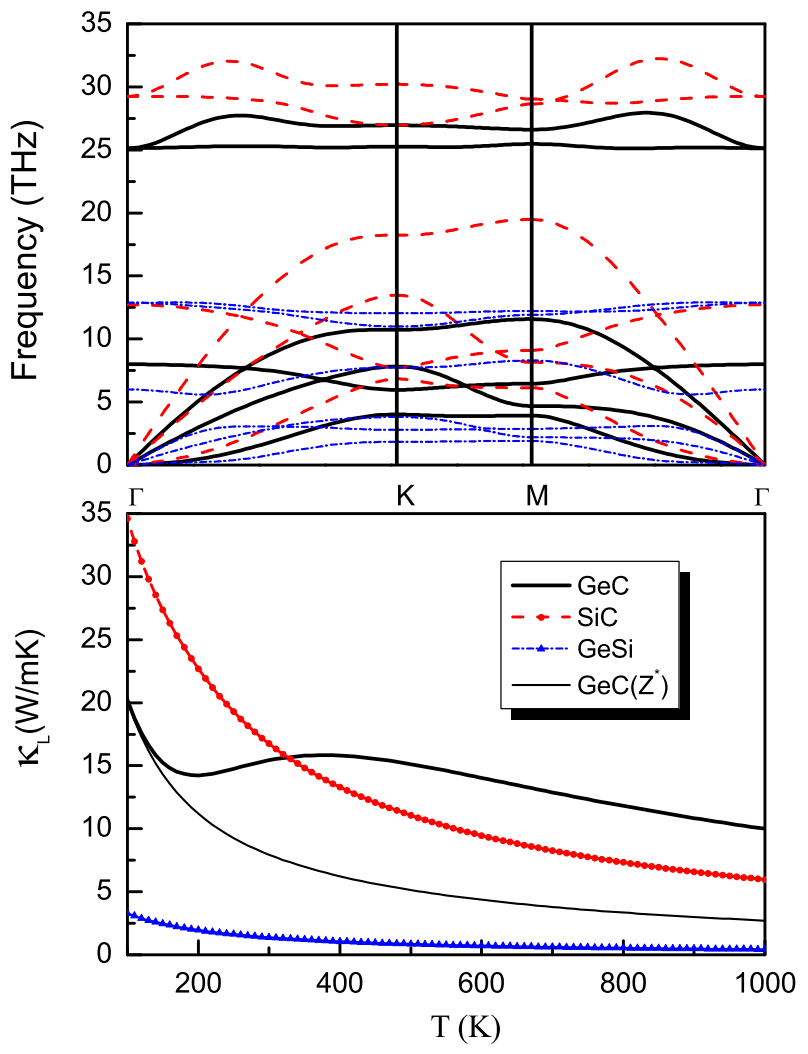

FIG. 9. (Color online)Left: phonon band structures of monolayer $\mathrm{GeC}, \mathrm{SiC}$ and $\mathrm{GeSi}$ without $\varepsilon$ and $Z^{*}$; Right: The $\kappa_{L}$ of monolayer $\mathrm{GeC}$ (without and with $\varepsilon$ and $Z^{*}$ ), SiC and GeSi.

tween with and without $\varepsilon$ and $Z^{*}$. However, for LO and TO branches, the group velocities and phonon lifetimes with $\varepsilon$ and $Z^{*}$ are smaller than ones without $\varepsilon$ and $Z^{*}$, which dramatically reduces contribution to $\kappa_{L}$ from LO and TO branches, and then the normal $\kappa_{L} \sim 1 / T$ relationship is observed in GeC monolayer.

Finally, the phonon-isotope scattering is consider on $\kappa_{L}$, based on the formula proposed by Shin-ichiro Tamura ${ }^{36}$. The related $\kappa_{L}$ with or without $\varepsilon, Z^{*}$ and isotope are plotted in Figure 8. Without inclusion of $\varepsilon$ and $Z^{*}$, it is clearly seen that the phonon-isotope scattering can also markedly remove $\kappa_{L} \sim T$ relationship. However, considering $\varepsilon$ and $Z^{*}$, the phonon-isotope scattering has little effects on $\kappa_{L}$ of monolayer GeC. In nanoscale devices, the residual strain usually exists, and the substrate is needed in real applications. Even if the $\varepsilon$ and $Z^{*}$ are neglected, the anomalous temperature dependence of $\kappa_{L}$ should depend on the strain and specific substrate.

\section{DISCUSSION AND CONCLUSION}

It is interesting to compare phonon transports of monolayer $\mathrm{GeC}$ with ones of $\mathrm{SiC}$ and $\mathrm{GeSi}$ monolayers. The monolayer $\mathrm{SiC}$ has also a perfect planar hexagonal honeycomb structure, but monolayer GeSi has a buckled struc- 
ture due to the decrease in the overlap between the $p_{z}$ orbitals $^{25}$. The phonon band structures and $\kappa_{L}$ of monolayer $\mathrm{GeC}, \mathrm{SiC}$ and $\mathrm{GeSi}$ without $\varepsilon$ and $Z^{*}$ are plotted in Figure 9. The $\varepsilon$ and $Z^{*}$ produce little effects on $\kappa_{L}$ of monolayer $\mathrm{SiC}$ and $\mathrm{GeSi}$, so only the $\kappa_{L}$ of monolayer $\mathrm{GeC}$ with $\varepsilon$ and $Z^{*}$ is shown in Figure 9 . It is clearly seen that anomalous temperature dependence of $\kappa_{L}$ is absent in monolayer $\mathrm{SiC}$ and $\mathrm{GeSi}$. These can be understood by their phonon dispersions. There is a huge phonon band gap of $13.52 \mathrm{THz}$ (larger than width of acoustic branches [11.61 THz]) in monolayer $\mathrm{GeC}$, which can weaken the scattering between acoustic and high frequency optical phonon modes, producing very large phonon lifetimes of LO and TO branches. For monolayer SiC, a phonon band gap of $7.47 \mathrm{THz}$ is observed (smaller than width of acoustic branches [19.48 THz]), and the LO and TO modes can be effectively scattered with acoustic modes, leading to short phonon lifetimes of LO and TO branches. In monolayer $\mathrm{GeSi}$, there is a very large gap of $7.17 \mathrm{THz}$ (larger than width of acoustic branches [3.81 THz]) between LO/TO and acoustic branches, but $\mathrm{ZO}$ branch is in the gap, which provide effective scattering channels for LO and TO branches, giving rise to short phonon lifetimes. When considering $\varepsilon$ and $Z^{*}$, the order of $\kappa_{L}$ is $\mathrm{GeSi}<\mathrm{GeC}<\mathrm{SiC}$, which is consistent with their atomic mass.
In summary, the phonon transports of monolayer $\mathrm{GeC}$ are investigated by the first-principles calculations and semiclassical Boltzmann transport theory. When neglecting $\varepsilon$ and $Z^{*}$, monolayer GeC possesses anomalously linear temperature dependent $\kappa_{L}$, which is different from the commonly established $\kappa_{L} \sim 1 / T$ relationship. The large deviation is because the contribution to $\kappa_{L}$ from LO and TO branches increases with increasing temperature, and eventually dominates the $\kappa_{L}$ with $T$ being larger than $300 \mathrm{~K}$. However, considering $\varepsilon$ and $Z^{*}$, the common $\kappa_{L} \sim 1 / T$ relationship is observed by reduced group velocities and phonon lifetimes of $\mathrm{LO}$ and TO branches. It is found that the phonon-isotope scattering can also weaken anomalously linear temperature dependent $\kappa_{L}$ in monolayer GeC. This work presents a comprehensive understanding of the phonon transports of monolayer $\mathrm{GeC}$, and sheds light on further studies of phonon transports of other $2 \mathrm{D}$ materials.

\section{ACKNOWLEDGMENTS}

This work is supported by the National Natural Science Foundation of China (Grant No.11404391). We are grateful to the Advanced Analysis and Computation Center of CUMT for the award of CPU hours to accomplish this work.
${ }^{1}$ K. S. Novoselov, A. K. Geim, S. V. Morozov, D. Jiang, Y. Zhang, S. V. Dubonos, I. V. Grigorieva and A. A. Firsov, Science 306, 666 (2004).

2 M. Chhowalla, H. S. Shin, G. Eda, L. J. Li, K. P. Loh and H. Zhang, Nature Chemistry 5, 263 (2013).

${ }^{3}$ R. X. Fei, W. B. Li, J. Li and L. Yang, Appl. Phys. Lett. 107, 173104 (2015).

${ }^{4}$ S. Ghatak, A. N. Pal and A. Ghosh, Acs Nano 5, 7707 (2011).

5 B. Radisavljevic, A. Radenovic, J. Brivio, V. Giacometti and A. Kis, Nature Nanotechnology 6, 147 (2011).

${ }^{6}$ X. Zong et al. J. Am. Chem. Soc. 130, 7176 (2008).

7 J. P. Ji, X. F. Song, J. Z. Liu et al., Nat. Commun. 7, 13352 (2016).

8 Z. Y. Al Balushi, K. Wang, R. K. Ghosh, R. A. Vilá, S. M. Eichfeld, J. D. Caldwell, X. Qin, Y.-C. Lin, P. A. DeSario, G. Stone, S. Subramanian, D. F. Paul, R. M. Wallace, S. Datta, J. M. Redwing and J. A. Robinson, Nat. Mater. 15, 1166 (2016).

9 M. Topsakal, S. Cahangirov, E. Bekaroglu and S. Ciraci, Phys. Rev. B 80, 235119 (2009).

10 Z. Yan, G. X. Liu, J. M. Khan and A. A. Balandin, Nat. Commun. 3, 827 (2012).

11 A. M. Marconnet, M. A. Panzer and K. E. Goodson, Reviews of Modern Physics 85, 1295, (2013).

12 A. I. Hochbaum, R. Chen, R. D. Delgado, W. Liang, E. C. Garnett, M. Najarian, A. Majumdar and P. Yang, Nature 451, 163 (2008).

13 G. Qin, Z. Qin, W.-Z. Fang, L.-C. Zhang, S.-Y. Yue, Q.-B. Yan, M. Hu and G. Su, Nanoscale, 8, 11306 (2016).
14 B. Peng, H. Zhang, H. Z. Shao, Y. F. Xu, G. Ni, R. J. Zhang and H. Y. Zhu, Phys. Rev. B 94, 245420 (2016).

15 D. C. Zhang, A. X. Zhang, S. D. Guo and Y. F. Duan, RSC Adv. 7, 24537 (2017).

16 B. Peng, D. Q. Zhang, H. Zhang, H. Z. Shao, G. Ni, Y. Y. Zhu and H. Y. Zhu, Nanoscale 9, 7397 (2017).

17 X. K. Gu and R. G. Yang, Appl. Phys. Lett. 105, 131903 (2014).

18 S. D. Guo, Phys. Chem. Chem. Phys. 20, 7236 (2018).

19 A. X. Zhang, J. T. Liu, S. D. Guo and H. C. Li, Phys. Chem. Chem. Phys. 19, 14520 (2017).

20 Y. D. Kuang, L. Lindsay, S. Q. Shic and G. P. Zheng, Nanoscale 8, 3760 (2016).

${ }^{21}$ H. K. Liu, G. Z. Qin, Y. Lin and M. Hu, Nano Lett. 16, 3831 (2016).

${ }^{22}$ H. M. Wang, G. Z. Qin, G. J. Li, Q. Wang and M. Hu, Phys. Chem. Chem. Phys. 19, 12882 (2017).

23 Z. Z. Qin, G. Z. Qin, X. Zuo, Z. H. Xiong and M. Hu, Nanoscale 9, 4295 (2017).

24 S. D. Guo and J. T. Liu, arXiv:1706.01025 (2017).

25 H. Sahin, S. Cahangirov, M. Topsakal, E. Bekaroglu, E. Akturk, R. T. Senger, and S. Ciraci, Phys. Rev. B 80, 155453 (2009).

26 G. Kresse, J. Non-Cryst. Solids 193, 222 (1995).

27 G. Kresse and J. Furthmüller, Comput. Mater. Sci. 6, 15 (1996).

28 J. P. Perdew, K. Burke and M. Ernzerhof, Phys. Rev. Lett. 77, 3865 (1996).

29 G. Kresse and D. Joubert, Phys. Rev. B 59, 1758 (1999).

30 A. Togo, L. Chaput and I. Tanaka, Phys. Rev. B 91, 
094306 (2015).

31 A. Togo, F. Oba, and I. Tanaka, Phys. Rev. B 78, 134106 (2008).

32 X. F. Wu, V. Varshney et al., Chem. Phys. Lett. 669, 233 (2017).

${ }^{33}$ R. C. Andrew, R. E. Mapasha, A. M. Ukpong and N.
Chetty, Phys. Rev. B 85, 125428 (2012).

34 E. Mariani and F. V. Oppen, Phys. Rev. Lett. 100, 076801 (2008).

35 J. Carrete, W. Li, L. Lindsay, D. A. Broido, L. J. Gallego and N. Mingo, Mater. Res. Lett. 4, 204 (2016).

36 S.I. Tamura, Phys. Rev. B, 27, 858 (1983). 